\title{
Marco de referencia para reflexionar sobre la implementación del Acuerdo de Paz, desde el enfoque de equidad de género*
}

Recibido: 25 de septiembre del 2018 • Aprobado: 23 de octubre del 2018

https://doi.org/10.22395/ojum.v17n35a3

Elvigia Cardona Zuleta*

\begin{abstract}
RESUMEN
El presente artículo presenta el marco de referencia para reflexionar sobre el estado de la implementación del Acuerdo final para la terminación del conflicto y la construcción de una paz estable y duradera (Acuerdo de Paz) firmado por el Gobierno Colombiano y las FARC-EP en octubre del 2016, en lo que respecta a la inclusión del enfoque de género, en el entendido de que las partes en este conflicto incluyen a la sociedad colombiana en su conjunto, y de que como ciudadanos es nuestro deber velar para que, tanto en la producción normativa como en la interpretación se tenga presente el enfoque de género; esto supondría una conquista en términos de la promoción de los derechos de las mujeres, niñas y grupos LGBTIQ y en especial del derecho a una vida libre de violencias.

Palabras clave: acuerdos de paz; enfoque de equidad de género; derechos humanos; acciones afirmativas.
\end{abstract}

Artículo de avance de investigación del proyecto en curso denominado "Implementación de los Acuerdos de Paz desde un enfoque de equidad de género para las mujeres" iniciado en mayo del 2018, cuya investigadora principal es Elvigia Cardona Zuleta. El proyecto ha sido financiado por la Convocatoria Paz y Posconflicto 2018-2020 de la Facultad de Derecho y Ciencias Políticas de la Universidad de Antioquia. Acta 14 del Comité Científico de Investigaciones del 5 de septiembre del 2018 e inscrita ante CODI con el número 2018-22790.

." Abogada de la Universidad de Antioquia, Medellín, Colombia y magíster en Educación de la misma universidad. Profesora e investigadora de dicha institución, adscrita al Grupo de Investigación Derecho y Sociedad, Medellín. Colombia. Correo electrónico: elvigia.cardona@udea.edu.co Orcid: https://orcid.org/0000-0002-2486-6867 


\section{Reference Framework to Reflect on the Peace Agreement Implementation from a Gender Equity Approach}

\section{ABSTRACT}

This paper presents a reference framework to reflect on the implementation condition of the Final agreement for the termination of the conflict and the construction of a stable and lasting peace (Peace Agreement) signed by the Colombian Government and the FARC-EP in October of 2016, focusing on the inclusion of the gender approach. This on the understanding that the parties in conflict include the Colombian society as a whole, and that as citizens, it is our duty to ensure that both in the normative production, as in its interpretation, the gender approach is taken into account. It would represent a conquest in the promotion of the rights of women, girls and LGBTIO groups, and especially for the right to live free of violence.

Keywords: peace agreements; gender equity approach; human rights; affirmative actions.

\section{Quadro de referência para refletir sobre a implementação do Acordo de Paz a partir da abordagem de igualdade de gênero}

\section{RESUMO}

O presente artigo apresenta o quadro de referência para refletir sobre o estado da implementação do Acordo final para a terminação do conflito e para a construção de uma paz estável e duradoura (Acordo de Paz), assinado pelo Governo colombiano e as FARC-EP em outubro de 2016. No que diz respeito à inclusão da abordagem de gênero, entende-se que as partes nesse conflito incluem a sociedade colombiana em seu conjunto e que, como cidadãos, é nosso dever zelar para que a abordagem de gênero esteja presente tanto na produção normativa quanto na interpretação. Isso suporia uma conquista em termos da promoção dos direitos das mulheres, meninas e grupos LGBTIQ e, em especial, do direito a uma vida livre de violências.

Palavras-chave: acordos de paz; abordagem de igualdade de gênero; direitos humanos; ações afirmativas. 


\section{INTRODUCCIÓN}

El Acuerdo final para la terminación del conflicto y la construcción de una paz estable y duradera (Acuerdo de Paz, 2016) ${ }^{1}$ firmado por el Gobierno Colombiano y las FARC-EP en octubre del 2016, presenta un reto para el Estado, las instituciones educativas y en general para la sociedad, puesto que será necesaria su veeduría de la implementación del acuerdo, las acciones afirmativas ${ }^{2}$ y los compromisos adquiridos por los actores. Sin embargo, no puede dejarse de lado que la inclusión de un capítulo dedicado al enfoque de igualdad de género y la conformación de una Instancia Especial para contribuir a garantizar el enfoque de género en la implementación del acuerdo final ${ }^{3}$, requiere cambiar la

1 Aún cuando las normas de citación APA señalan que las referencias dentro del cuerpo del texto deben indicar entre paréntesis los apellidos de los autores y la fecha, en este trabajo, en lo que respecta al caso del Acuerdo final para la terminación del conflicto y la construcción de una paz estable $y$ duradera, opto por abreviar entre paréntesis de la siguiente manera: (Acuerdo de Paz, 2016), con el argumento de que si bien el Gobierno de Colombia y las FARC-EP son las que firman el acuerdo, este incumbe a toda Colombia, por ende su implementación es un asunto que compete al conjunto de la sociedad colombiana, independientemente de la corriente política o sector social. Considero que esta decisión facilita la apropiación individual del proceso.

2 La acción afirmativa es una figura constitucionalmente aceptada que da cumplimiento al derecho a la igualdad material en pos de alcanzar la equidad con medidas legales, administrativas, políticas o prácticas; pretende eliminar factores discriminatorios históricos que impiden a hombres y mujeres compartir en un plano de igualdad real. Dichas desigualdades, además de estar vinculadas a la condición de ser hombre o mujer, incrementan al cruzarse con otras características, tales como raza, etnia, identidad de género, orientación sexual, la discapacidad, condición socioeconómica, nivel educativo, edad, entre otros.

3 Instancia Especial incluida en el punto 6.1. Mecanismos de Implementación y Verificación, literal d. (...) Instancia Especial conformada por representantes de 6 organizaciones de mujeres colombianas nacionales y territoriales perspectiva, los referentes teóricos y jurídicos con los cuales se analizan las decisiones de las diferentes ramas del poder y que inciden en la implementación, debido a que representa un:

\section{[...] hito en materia de construc- ción de paz a nivel internacional ya que ubica los derechos de las mujeres y a la igualdad de género en el centro del Acuerdo. Es una innovación del Acuerdo colombiano. En el texto se crean medidas y disposiciones concre- tas para promover la igualdad de derechos, la participación activa y la representación equitativa de las mujeres en la construcción de paz. (Instituto Kroc, 2017, p. 12)}

Sin embargo, en el texto final del Acuerdo de Paz influyeron las presiones en contra de su firma por parte de un sector de la sociedad colombiana, previo al plebiscito del 2 de octubre del 2016 y que se encuentran asociadas a la campaña del "no". Estas fueron determinantes para la modificación de algunos apartes del documento, específicamente los que hacían referencia al enfoque de género propuesto inicialmente. La protesta derivó de debates centrados en señalar que existía en el Acuerdo de Paz una "ideología de género", que afectaba la concepción y valores tradicionales asignados a la familia. 
La Fundación Ideas para la Paz (FIP), indica que se realizaron cambios cosméticos al enfoque de género en el texto final de los Acuerdos de Paz:

\begin{abstract}
La FIP identificó que el 50\% de las propuestas sobre enfoque de género fueron presentadas por el exprocurador general Alejandro Ordóñez y las iglesias cristianas. Estas buscaban limitar el alcance del enfoque solo al reconocimiento de los derechos de las mujeres. Por el contrario, las propuestas de organizaciones de mujeres y LGBTI, pedían que se incluyera a población con identidades sexuales diversas. Por su parte, el expresidente Andrés Pastrana y la líder conservadora Marta Lucía Ramírez, proponían que el nuevo Acuerdo se concentrara en reconocer a las mujeres como víctimas particulares del conflicto armado. (Mazzoldi y Cuesta, 2017, p. 1)
\end{abstract}

Para puntualizar, el Acuerdo Final "no incluyó un 'enfoque de género', sino uno centrado en los derechos de las mujeres y las medidas afirmativas; las menciones de 'diversidad sexual', 'orientación sexual' e 'identidad de género' fueron eliminadas y reemplazadas por un 'enfoque familiar'" (OIM, 2018, p. 1), toda vez que la población LGBTIO (lesbianas, gays, bisexuales, transexuales, intersexuales y queer) se incluyó en los grupos vulnerables. (OIM, 2018).

Así las cosas, se evidencian diferencias de orden conceptual al nombrar el enfoque de género en los apartes del texto final del Acuerdo de Paz; a veces se aborda como igualdad entre hombres y mujeres, mientras que en otros apartados el enfoque de género tiene un significado que directamente atañe a las mujeres, con expresiones como: "reconocimiento de las mujeres como ciudadanas autónomas" (Acuerdo de Paz, 2016, p. 12). En otros apartes el enfoque de género se usa como si fuese un adjetivo más: "enfoque territorial, diferencial y de género" (Acuerdo de Paz, 2016, pp. 26, 39-40, entre muchas otras ocasiones). Y finalmente, se incluyen referencias que textualmente se denominan "medidas afirmativas" (pp. $30,35,51,55,80,92,119$, entre otras) que están ligadas al concepto de equidad, en el sentido de la necesidad de una igualdad real y efectiva.

En palabras de Catherine Walsh, "nombrar, también es luchar" (2010, p. 209), por eso en la investigación en curso, de la que se desprende este artículo de avance, la pregunta orientadora es "¿cómo verificar la implementación de las acciones afirmativas desde un enfoque de equidad de género para las mujeres, en el marco del Acuerdo final para la terminación del conflicto y la construcción de una paz estable y duradera, firmado por el Gobierno Colombiano y las FARC-EP en octubre del 2016 (Acuerdos de Paz) (2017-2018)?". El objetivo del presente avance es describir el marco referencial para reflexionar sobre la implementación del Acuerdo de Paz desde un enfoque de equidad de género para las mujeres.

En este sentido, es importante aclarar que se parte de un enfoque de equidad de género para las mujeres, no porque se desconozca 
la existencia de fuertes discriminaciones a grupos vulnerables como la población LGBTIQ, sino por dos motivos: i) la forma difusa de nombrar hace que se dificulte la comprensión del alcance de las medidas afirmativas propuestas, pues no basta con que se diga que son medidas para lograr la igualdad real y efectiva dirigidas a las mujeres, ni que se incluyan palabras como igualdad entre hombres y mujeres, para inferir la existencia de decisiones políticas y jurídicas que concreten la implementación con perspectiva de género; ii) la renuencia tanto de las instituciones estatales como de la misma academia para transversalizar la perspectiva de género y reconocer explícitamente las asimetrías de poder, niveles de exclusión y discriminación estructurales, persistentes en la cultura colombiana en el trato de hombres y mujeres, que contribuyen a la construcción, normalización y reproducción de patrones de exclusión basados en los roles, prejuicios y estereotipos sexogenéricos, que finalmente, en el caso de las mujeres, constituirían una barrera al momento de implementar las acciones afirmativas propuestas en los Acuerdos de Paz que algunos sectores perciben como desproporcionadas o innecesarias.

En efecto, la reafirmación y reconocimiento que hace el Acuerdo de Paz del liderazgo y la necesaria participación de las mujeres en la prevención y solución de los conflictos (Lopera et al., 2018) señala un camino mediante acciones afirmativas que permite superar las desigualdades estructurales y culturales que han llevado a mujeres y niñas a experimentar - de manera diferenciada y desproporcio- nada - los embates de la guerra y los diversos tipos de violencia; entre ellos, físicos, psicológicos, sexuales, patrimoniales e institucionales (Ley 1257 de 2008, Convención de la Cedaw y Belem do Pará), tanto en el marco de los conflictos vividos en sus territorios y perpetrados por diversos actores (armados y no armados, legales e ilegales), como en su condición de mujeres y niñas desplazadas y víctimas de violencias.

Esto da lugar a que en el Acuerdo de Paz se evidencie la presencia de cerca de ciento veintidós (122) acciones afirmativas dirigidas a materializar los derechos de las mujeres en un plano de equidad (Pineda, 2017; Instituto Kroc, 2017). Con estas se pretende reconocer la existencia la discriminación estructural, la cual se cristaliza en prejuicios y estereotipos que impiden a las mujeres y niñas el goce efectivo y en condiciones de equidad y justicia sus derechos.

Algunas de esta medidas se dirigen a garantizar el acceso de las mujeres en condiciones de igualdad a un sistema de salud integral (Acuerdo de Paz, 2016, p. 26), a la educación (p. 27), al Sistema Integral de Seguridad para el Ejercicio de la Política (p. 39), a la planeación democrática y participativa (p. 50); a la participación política y ciudadana (p. 52), a una administración de justicia libre de prejuicios (p. 79), a garantías de reincorporación (p. 81); a la formación de funcionarios públicos y policía sobre violencias y justicia de género (p. 84), a la solución al problema de drogas ilícitas y 
su relación con violencias sexuales y de género (p. 98), al Sistema Integral de Verdad, Justicia, Reparación y No Repetición (p. 127), entre muchas otras referencias que serán objeto de revisión en esta investigación. Lo anterior porque se considera que, sumado a las dificultades del lenguaje, las posibilidades de que se reviertan los derechos alcanzados por las mujeres en el marco de la construcción de paz estable y duradera debido a una deficiente implementación, por la falta de asignación de recursos suficientes o por la falta de criterios conceptuales al utilizar el enfoque de género, como una muletilla o adjetivo más, es latente.

Adicionalmente, la decisión tomada por el Consejo de Estado el 7 de marzo del 2018, de suspender provisionalmente la función otorgada a la Comisión de Seguimiento, Impulso y Verificación a la Implementación del Acuerdo Final (CSIVI) por el Decreto 1995 del 7 de diciembre de 2016, en lo que se refiere a

\begin{abstract}
Constatar que el contenido de todos los proyectos de decreto, ley o acto legislativo que sean necesarios para implementar el Acuerdo Final, correspondan a lo acordado antes de que sean expedidos por el Presidente de la República o presentados ante el Congreso, según sea el caso.
\end{abstract}

Es decir que la función otorgada a la instancia especial para garantizar el enfoque de género en la implementación del acuerdo, se reduce a "proponer", por considerar que su injerencia constituye en "un desbordamiento que afecta la competencia de las instituciones reconocidas constitucionalmente" (El Tiempo, 2018). Se configura así un retroceso en la implementación, lo que exige que las organizaciones no gubernamentales y la academia asuman su función de veeduría para la construcción de una sociedad equitativa y justa.

En este escenario se requiere que las mujeres y las niñas superemos la posición de víctimas y asumamos el rol como ciudadanas constructoras de paz, al igual que le corresponde a las instituciones y a la sociedad, entender que la aspiración de lograr la equidad de género para las mujeres va más allá de la implementación del acuerdo, y que como ciudadanos (sin importar el género), se requiere estar atentos a la implementación, con indicadores, evaluaciones y la posibilidad de exigir por las vías jurídicas y políticas la puesta en marcha de los compromisos pactados.

En términos metodológicos esta investigación es de corte cualitativo y se enmarca en el paradigma crítico, desde el que se cuestiona la manera en que "la investigación cualitativa, en casi todas sus formas (observación, participación, entrevistas, etnografía), funciona como una metáfora del conocimiento, el poder y la verdad coloniales" (Denzin y Lincoln, 2012, p. 43), por lo que se propende por epistemologías feministas y enfoques de género que permitan realizar una investigación situada, contextualizada y que reconoce las apuestas políticas y los valores presentes de la investigadora y 
estudiantes en formación del Semillero Género y Derecho como sujetos que participan, construyen y resignifican el mundo en el que viven.

De manera acorde, se exige a "los académicos que demuestren sus intenciones éticas y políticas, es decir, que se conviertan en un intelectual transformador, un activista, un defensor, un emancipador, un investigador comprometido con el cambio y la justicia social." (Denzin y Lincoln, 2012, p. 42). Es así que esta investigación en su conjunto es un escenario de formación investigativa y a su vez, una apuesta política por generar sensibilidad crítica frente al sistema sexo-género.

Para efectos del objetivo mencionado, se recurre a la revisión documental y se consultan los informes disponibles hasta agosto del 2018 provenientes de organizaciones encargadas de producirlos. Para ello, se diseñó como instrumento de recolección y sistematización de la información una ficha que permite recabar información con los pasos propuestos por Alda Facio (1992) para abordar un texto legal -estos se explican en el siguiente apartado-, y así realizar un análisis y una lectura cruzada.

Finalmente y para darle orden a este artículo, en primer lugar se señalan algunos de los referentes teóricos y normativos que permiten situarse en el enfoque de equidad de género para las mujeres; en segundo lugar se presentan informes especializados sobre el avance de la implementación del Acuerdo de Paz, a fin de verificar en qué va la transversalización del enfoque de género; en tercer lugar se aborda el Plan Marco de Implementación (PMI) que será el referente a 15 años. A modo de reflexiones preliminares se concluye con unas intuiciones/hallazgos de esta primera etapa de la investigación.

\section{SOBRE EL ENFOQUE DE EQUIDAD DE GÉNERO PARA LAS MUJERES}

Situar el punto de partida en el enfoque de equidad de género para las mujeres supone en primer lugar, ubicar teórica y normativamente la categoría género en el fenómeno jurídico y, en segundo lugar, explicitar los alcances y elementos de la figura denominada acción afirmativa, tareas poco pacíficas según las múltiples visiones y discursos que imperan en la actualidad en el gobierno, las instituciones y la academia. Una acción afirmativa faculta al gobierno a expedir leyes, decretos, políticas públicas, directivas, entre otras, que en un primer momento pueden aparentar ser desiguales, pero que en la práctica alcanzan la igualdad real y efectiva. Es decir que estas son equitativas, en contraposición con aquellas normas que inicialmente parecen favorecen la igualdad o aparentan neutralidad, pero que en la práctica diaria ahondan la discriminación y exclusión de la mujer -en este caso particular-, en ámbitos familiares y públicos, acceso a sus derechos y participación política.

Con respecto a la ubicación teórica del enfoque de equidad de género, es preciso señalar que es un enfoque teórico y también un 
enfoque metodológico, estas discusiones se abordan a partir de los trabajos de Alda Facio (1992), quien propone realizar un análisis del fenómeno jurídico, en el cual el componente político-cultural juega un papel determinante en la producción, interpretación y aplicación normativa:

\begin{abstract}
El fenómeno jurídico no se reduce a las leyes formalmente generadas (componente formal-sustantivo), sino que se compone también de las leyes que se forman a través de la administración e interpretación de esas leyes formalmente generadas (componente estructural) y también de las leyes que se forman a través de las costumbres, tradiciones, políticas, así como del conocimiento y uso que le dé la gente a las leyes formalmente promulgadas o formalmente interpretadas (componente político-cultural). (Facio, 1992, p. 53)
\end{abstract}

Es decir que es necesario entender que la producción, interpretación y aplicación normativa pasa por procesos culturales instalados como el androcentrismo, sexismo, machismo, discriminación, exclusión, entre otros, los cuales están presentes en la cultura jurídica. Es por eso es necesario develar, para transformar y proponer otros instrumentos que permitan hacer una lectura que integre el enfoque de equidad de género en las actividades diarias de los funcionarios (independientemente de su género) en todos los niveles y ramas del poder público.

En este sentido, Alda Facio propone una metodología de seis pasos para realizar un análisis del texto legal, los cuales se aplican a continuación para hacer una lectura de todas las normas y documentos expedidos durante el 2018, dirigidos a la implementación del Acuerdo de Paz. Estos pasos los presenta Facio en su libro Cuando el género suena, cambios trae: una metodología para el análisis de género del fenómeno legal, del cual se hace una cita extensa que permite dimensionar el componente teórico-metodológico del desarrollo de la investigación en curso:

Paso 1. Tomar conciencia a partir de la experiencia personal, de la subordinación del género femenino al masculino. (p. 75)

Paso 2: Se trata de profundizar en la comprensión de lo que es el sexismo y las formas en que se manifiesta, identificando y cuestionando los elementos de la doctrina jurídica, de los principios y fundamentos legales y de las investigaciones que fundamentan esos principios y esas doctrinas, que excluyen, invisibilizan o subordinan a las mujeres. (p. 77)

Paso 3: Identificar cuál es la mujer que la ley está contemplando como "el otro" del paradigma de ser humano que es el hombre y analizar sus efectos en las mujeres de distintos sectores, razas, orientaciones sexuales, discapacidades visibles, edades, etc. (p. 95)

Paso 4: Buscar culál 1 es la concepción de "mujer" que sirve de sustento al texto para encontrar soluciones prácticas a la exclusión, los problemas y necesidades de las mujeres que no impliquen 
la institucionalización de la desigualdad. (p. 96)

Paso 5: Analizar el texto tomando en cuenta los otros dos componentes. Es decir, si es un proyecto ley (componente formal normativo), analizar el contenido y efectos que tendrá en los componentes políticocultural y estructural. Si es una doctrina jurídica, (componente político-cultural) ver cómo o si se ha infiltrado en el componente formal normativo y qué influencia tiene en el estructural, etc. O si es un contexto legal, es decir, si se está partiendo de una realidad concreta de una mujer o un grupo de mujeres, preguntarse cuáles son sus problemas, intereses y necesidades legales y luego ver si éstas [sic] se encuentran reflejadas en los componentes. (p. 99)

Paso 6: Colectivizar el análisis, no sólo [sic] para que sea enriquecido por mujeres (y hombres conscientes) de distintos sectores a la vez que se hace educación legal popular, sino más importante aún, para continuar el proceso de conscientización que es, como lo he venido diciendo, el paso previo a cualquier análisis de un texto legal, ya que sin la toma de conciencia de que las mujeres por nuestro sexo, somos subordinadas y discriminadas, ni siquiera se puede iniciar un cuestionamiento de un sistema legal desde una perspectiva de género. (p. 109)

De esta forma, a medida que avanza la investigación en curso, se han desarrollado fichas de lectura que permiten categorizar los documentos y normatividad expedida, se hace un análisis inicial de nuestras concepciones, puesto que al momento de iniciar los encuentros en el equipo de investigación ${ }^{4}$ se advierte, por ejemplo, la dificultad que supone tomar conciencia de que, como mujeres, tenemos una condición subordinada y que como hombres, a veces se gozan de privilegios que hasta el momento no habíamos percibido o se tomaban como una situación externa teórica, alejada de la cotidianidad.

A manera de recapitulación se establece que el enfoque teórico se sitúa en la equidad en tanto es una aspiración para lograr la justicia, lo cual requiere acciones y recursos que faciliten el acceso y garanticen la efectividad de los derechos humanos. En este caso en específico, se parte de un análisis de la situación de las mujeres que, representan un 52\% de la población mundial.

Ahora bien, en cuanto a los referentes normativos que permiten establecer ese lugar de enunciación denominado enfoque de equidad de género para las mujeres - el cual tiene un carácter institucional y cuenta con un marco normativo que permite explicar su importancia en términos de las posibilidades reales de justiciabilidad y exigencia gubernamental-, cabe explicitar que: Colombia incluyó en el ordenamiento jurídico las obligaciones

4 La Convocatoria Paz y Posconflicto 2018-2020, incluía entre sus requisitos la vinculación de dos (2) estudiantes de pregrado en formación. A partir del mes de septiembre del 2018 se conformó el equipo de investigación con cinco (5) estudiantes de pregrado, cuatro mujeres y un hombre, adscritos al Semillero Género y Derecho, con quienes se inició la formación inicial sobre las implicaciones de partir del enfoque de género para las mujeres. 
internacionales adquiridas frente a la Convención sobre la Eliminación de Todas las Formas de Discriminación contra las Mujeres (Cedaw por sus siglas en inglés). Dicha convención fue expedida en 1979 y entro en vigor en 1981 y en su artículo primero señala:

\begin{abstract}
A los efectos de la presente Convención, la expresión "discriminación contra la mujer" denotará toda distinción, exclusión a restricción basada en el sexo que tenga por objeto o por resultado menoscabar o anular el reconocimiento, goce o ejercicio por la mujer, independientemente de su estado civil, sobre la base de la igualdad del hombre y la mujer, de los derechos humanos y las libertades fundamentales en las esferas política, económica, social, cultural y civil o en cualquier otra esfera. (ONU, 1979)
\end{abstract}

La Cedaw toma como base la igualdad de hombre y mujer, su relación con el goce o ejercicio de los derechos, lo cual supone una concepción de igualdad material e incluye todas las esferas de desempeño de las mujeres en las cuales se presentaban fuertes discriminaciones, poniendo de manifiesto que, además de la esfera privada, existe una relación con espacios de carácter público en la cual se reconoce que las mujeres no están en el mismo plano de igualdad que los hombres.

Posteriormente, en 1994 la Organización de los Estados Americanos (OEA, OAS por sus siglas en inglés) organizó la Convención Interamericana para Prevenir, Sancionar y Erradicar la Violencia contra la Mujer que tuvo lugar en Belem Do Pará (también conocida como Convención de Belem Do Pará). En su artículo $4^{\circ}$ señala que: "Toda mujer tiene derecho al reconocimiento, goce, ejercicio y protección de todos los derechos humanos [...] f) El derecho a igualdad de protección ante la Ley y de la Ley" (OEA, 1995), en esta expresión normativa se conjuga la igualdad material y la igualdad de trato.

Estos dos referentes internacionales (Cedaw y Convención de Belem Do Pará) se concretan directamente en las siguientes normas: Ley 51 de 1981. Por medio de la cual se aprueba la "Convención sobre la eliminación de todas las formas de discriminación contra la mujer", adoptada por la Asamblea General de las Naciones Unidas el 18 de diciembre de 1979 y firmada en Copenhague el 17 de julio de 1980; Ley 248 de 1995. Por medio de la cual se aprueba la Convención Internacional para prevenir, sancionar y erradicar la violencia contra la mujer, suscrita en la ciudad de Belem Do Pará, Brasil, el 9 de junio de 1994; Ley 294 de 1996. Por la cual se desarrolla el artículo 42 de la Constitución Política y se dictan normas para prevenir, remediar y sancionar la violencia intrafamiliar, decretada por el Congreso de Colombia; Ley 1257 de 2008. Por la cual se dictan normas de sensibilización, prevención y sanción de formas de violencia y discriminación contra las mujeres, se reforman los Códigos Penal, de Procedimiento Penal, la Ley 294 de 1996 y se dictan otras disposiciones, decretada por el Congreso de Colombia. 
A la fecha (2018), estas normas siguen siendo inoperativas, nugatorias y carentes de contenido económico, formativo y político.

Adicionalmente, en Colombia, mediante la Ley 1450 de 2011. Plan de Desarrollo 20102014, se habla de la Política de Equidad de Género en los siguientes términos:

EQUIDAD DE GÉNERO. El
Gobierno Nacional adoptará
una política pública nacional
de Equidad de Género para ga-
rantizar los derechos humanos
integrales e interdependientes
de las mujeres y la igualdad de
género, teniendo en cuenta las
particularidades que afectan a
los grupos de población urbana
y rural, afrocolombiana, indígena,
campesina y Rom. La política
desarrollará planes específicos
que garanticen los derechos
de las mujeres en situación de
desplazamiento y el derecho de
las mujeres a una vida libre de
violencia. Esta política pública
será construida de manera par-
ticipativa bajo la coordinación
de la Alta Consejería para la
Equidad de la Mujer (Acpem), la
cualserá fortalecida institucional
y presupuestalmente para el
cumplimiento efectivo de sus
responsabilidades y funciones.
(Congreso de la República, 2017
Ley 1450 de 201l, art. 177, subra-
yado fuera de texto)

Esta norma ratifica la labor de la ya existente Consejería para la Equidad de las Mujeres y la eleva al rango de Alta Consejería Presidencial para la Equidad de la Mujer, pero en el segundo periodo presidencial de Juan Manuel Santos pasó nuevamente a ser parte de una de las Secretarías presidenciales. Desde allí, en el 2012 se diseñaron los lineamientos para la política pública nacional de equidad de género para las mujeres, los cuales presentan una orientación más marcada a que las mujeres experimenten "una vida libre de violencias" (Acpem, 2012, p. 8). El desarrollo y la expedición de los mismos recibió el acompañamiento de ONU Mujeres, Agencia Española de Cooperación Internacional (Aecid), Proyecto de Mujer de la Agencia Alemana de Cooperación (GIZ), Fondo de Población de las Naciones Unidas (Unfpa), Programa de Naciones Unidas para el Desarrollo (PNUD), Agencia de Naciones Unidas para Refugiados (Acnur), Programa Integral de Violencias de Género, Programa Ventana de Paz, Agencia del Desarrollo Internacional de los Estados Unidos (Usaid), Embajada de Canadá, Embajada Real de los Países Bajos, Embajada de Noruega y Embajada de Suecia.

Estos lineamientos de la política pública de equidad de género para las mujeres contienen un marco conceptual, principios orientadores, objetivos y estrategias que debe implementar el gobierno, ya que reconoce que:
Existe aún en el país una brecha considerable entre el reconoci- miento de derechos y el ejercicio de éstos [sic] para la mayoría de mujeres, especialmente para aquellas que enfrentan situacio- nes de especial vulnerabilidad o aquellas que evidencian la intersección de múltiples dis- 
criminaciones, generándoles una afectación desproporcionada y una relación inequitativa. (Acpem, 2012, p. 4)

Posteriormente, el documento Conpes (Consejo Nacional de Política Económica y Social) 161 de 2013 centrado en la Equidad de Género para las Mujeres, define:

En esta dirección, la Política Pública Nacional de Equidad de Género para las Mujeres, en sus lineamientos, incorpora como herramientas conceptuales el principio de igualdad y no discriminación, el análisis de género y el enfoque diferencial de derechos. Este sentido amplio del concepto de equidad, que le da nombre a esta política pública nacional, adopta las nociones de igualdad, no discriminación y diversidad, cuyo entrelazamiento permite definir la equidad como una igualdad orientada por las diferencias. Esto no significa que mujeres y hombres deban convertirse en iguales, sino que el Estado debe garantizar el acceso, goce y ejercicio en igualdad de oportunidades y capacidades para unos y otros. (DNP, 2013, p. 8)

Adicionalmente, en este se presenta el enfoque diferencial de derechos y la intersección de otras discriminaciones a las mujeres, el cual se traduce en lo siguiente:

Este enfoque se traduce en la utilización de procedimientos diferenciales para corregir desigualdades de partida; medidas no necesariamente iguales, pero conducentes a la igualdad de acceso en términos de derechos, beneficios, servicios y activos conocidas como acciones positivas o afirmativas. Éstas [sic] facilitan a los grupos de personas consideradas en desventaja, en este caso mujeres y grupos específicos de mujeres, el acceso a oportunidades y resultados, que buscan superar las brechas de desigualdad. (DNP, 2013, p. 9)

El Conpes 161 también contempla seis ejes temáticos: 1) construcción de paz y transformación cultural; 2) autonomía económica y acceso a activos; 3) participación en los escenarios de poder y toma de decisiones; 4) salud y derechos sexuales y reproductivos; 5) enfoque de género en la educación; 6) plan integral para garantizar a las mujeres una vida libre de violencias.

Finalmente, la Ley 1753 de 2015, Plan Nacional de Desarrollo 2015-2018, plantea lo siguiente al referirse a los principios que orientan el Plan:

Estos procesos deben tener un enfoque diferencial que promueva la garantía de derechos de comunidades étnicas, niños y adolescentes, población con discapacidad y la tercera edad; y por el imperativo de promover integralmente los derechos de las mujeres y la igualdad de género, desde una perspectiva interseccional (Congreso de la República, 2015, p. 46; citado en Alta Consejería Presidencial para la Equidad de la Mujer, 2012)

A partir de estas consideraciones se jus- 
tifica partir de un enfoque de equidad de género para las mujeres como eje central de las argumentaciones, ya que constituye una posibilidad real para la exigibilidad y justiciabilidad de los derechos de las mujeres. Además, permite la inclusión de categorías como la interculturalidad, la interseccionalidad y los enfoques diferenciados para abordar -en la práctica jurídica y política- la implementación del Acuerdo de Paz y adelantar acciones jurídicas y educativas que permitan la superación de la discriminación y exclusión normativa, atendiendo a las particularidades de las acciones afirmativas propuestas por el Acuerdo de Paz.

Ahora bien, frente a las acciones afirmativas es necesario partir de la Constitución Política de Colombia (1991), puesto que su artículo 13 sirve para ejemplarizar la manera en la cual se incluyen las diferentes acepciones de igualdad del ordenamiento jurídico colombiano, en primer lugar, en la expresión: "Todas las personas nacen libres e iguales ante la ley" (Asamblea Nacional Constituyente, ANC,1991, art. 13) en la cual el constituyente primario parte de una idea de igualdad de características que tiene que ver con la comparación frente a la categoría "personas". En segundo lugar, en la expresión: "recibirán la misma protección y trato de las autoridades" (ANC,1991, art. 13) en la cual emerge el concepto de igualdad de trato. Y en tercer lugar, en la expresión: "El Estado promoverá las condiciones para que la igualdad sea real y efectiva y adoptará medidas a favor de grupos discriminados o marginados" (ANC,1991, art. 13), en la que el constituyente presenta la idea de la igualdad material por vía de la protección especial del Estado, para alcanzar la equidad y la justicia social, ya que reconoce que hay unos grupos (en el caso de las mujeres, una población) discriminados o marginados que requieren especial atención.

Con la idea de las acciones afirmativas se busca corregir las desigualdades de partida o estructurales para garantizar posibilidades reales de ejercicio de los derechos para sectores de la población en situación de marginalidad o que manifiestan vulnerabilidad, en este caso las mujeres. Estas fueron propuestas por los movimientos feministas liberales en la década de los ochentas del siglo XX en Estados Unidos por autoras como Betty Friedan, como una alternativa para superar la desigualdad y discriminación (Cobos, 2014).

Por otro lado, con el cambio constitucional colombiano de 1991 y las presiones internacionales para dar cumplimento a las Convenciones del Sistema Universal de Derechos y el Sistema Interamericano de Derechos se crea un marco de referencia normativo general de protección a las mujeres en el que las acciones afirmativas se interpretan como una figura constitucionalmente aceptada que materializa el derecho a la igualdad material, en pos de alcanzar la equidad a través de medidas legales, económicas, administrativas, políticas o prácticas (Cedaw) para eliminar factores discriminatorios históricos que impiden 
a hombres y mujeres estar en un plano real de igualdad. Dichas desigualdades, además de estar vinculadas a la condición de ser hombre o mujer, incrementan al cruzarse con otros factores tales como raza, etnia, identidad de género, orientación sexual, discapacidad, condición socioeconómica, nivel educativo, edad, entre otros. Estas medidas o acciones son de carácter transitorio (hasta que se superen las situaciones de discriminación) y requieren de un contenido económico o de acciones concretas y reales que permitan hacer efectivos los derechos y alcanzar la equidad y la justicia social.

\section{INFORMES ESPECIALIZADOS EN EL SEGUIMIENTO A LA IMPLEMENTACIÓN DE LOS ACUERDOS DE PAZ}

Se encontraron los siguientes informes especializados sobre la implementación del Acuerdo de Paz que tratan específicamente la perspectiva o enfoque de género:

En primer lugar, están los Informes sobre el estado efectivo de la implementación del Acuerdo de Paz en Colombia elaborados por el Instituto Kroc de Estudios Internacionales de Paz de la Universidad de Notre Dame, encargados de realizar el apoyo técnico (Acuerdo de Paz, 2016, p. 212), no solo en términos del diseño de la metodología de seguimiento a la implementación, sino en la producción de informes que sean útiles para la toma de decisiones de las demás comisiones y grupos de trabajo, conforme se definen las funciones y mecanismos de verificación e implementación del
Acuerdo de Paz.

El primer informe, presentado en noviembre del 2017, desagrega el Acuerdo en una matriz con las 578 disposiciones que corresponden a 74 subtemas, 18 temas y 6 puntos del Acuerdo de Paz y de esta forma, constituye un insumo metodológico, ya que como señalan, su diseño permite medir la implementación en tiempo real:

1) el estado actual del progreso en la implementación del Acuerdo de Paz, el cual incluye enfoques transversales de derechos humanos, género, étnico y territorial; 2) patrones emergentes en la implementación, avances, dificultades, brechas y eventuales retrocesos; 3) lecturas sistémicas, secuenciales e interdependientes entre los diversos temas, subtemas y disposiciones del Acuerdo de Paz, los contextos en donde se implementa y el análisis de los posibles efectos en cascada (positivos o negativos) de la implementación del Acuerdo; y 4) datos comparativos relevantes que muestran las tendencias y la evolución con base en otros procesos de implementación. (Instituto Kroc, 2017, p. 4)

Adicionalmente, este informe marca la pauta en términos de identificación de la información necesaria para verificar el cumplimento en el corto, mediano y largo plazo mediante el uso de herramientas metodológicas que se pueden replicar eventualmente, tales como la identificación de "patrones emergentes en la implementación [...] lecturas sistemáticas, 
secuenciales e interdependientes entre los diversos temas y subtemas" (Instituto Kroc, 2017, p. 4) y la utilización de mapas mentales, formularios para el registro de información y bases de datos que faciliten el seguimiento y la comparación. Sin embargo, como lo señala el mismo informe, "La metodología acordada con la CSIVI para medir estos enfoques más amplios está en desarrollo" (Instituto Kroc, 2017, p. 12).

Posteriormente, en septiembre del 2018 el Instituto Kroc presentó el segundo informe para el periodo diciembre 2016 mayo 2018, en el que incluyó un capítulo sobre la transversalización del enfoque de género, señalando como principales dificultades: "recursos limitados para garantizar acciones afirmativas [...] falta de paridad para las mujeres en instancias técnicas y políticas" (Instituto Kroc, 2018, p. 268) . De esta manera, evidenció que de las 578 disposiciones del Acuerdo Final, al 31 de mayo del 2018, "no se ha iniciado el 30\% de los compromisos en el Acuerdo, el 31\% están mínimamente implementados, el 9\% ha alcanzado un nivel intermedio de implementación, y el $21 \%$ de los compromisos se han implementado completamente" (p. 269).

En segundo lugar, se encuentra el informe de gestión de la Instancia Especial para contribuir a garantizar el enfoque de género en la implementación del Acuerdo Final, la cual presentó en marzo de 2018 el primer informe de gestión de esta subcomisión para el periodo juliodiciembre de 2017. Esta instancia "fue creada con el objetivo de hacer veeduría y seguimiento, desde la sociedad civil, a la implementación [...] y garantizar el cumplimiento de las más de 100 medidas que contempla el Acuerdo Final con relación a los derechos de las mujeres y las personas LGBTI" (Instancia Especial para contribuir a garantizar el enfoque de género en la implementación del Acuerdo Final, 2018, p. 3) . En ella se señala como un logro, la creación de la octava representación "reconociendo la justa demanda de las mujeres afrocolombianas, raizales y palanqueras" (2018, p. 2) y rescatan la composición de la instancia que ha permitido el trabajo colectivo. Asimismo, y de manera específica, llaman la atención frente a los siguientes puntos que se han implementado sin el enfoque de género y de forma imperfecta, sin indicar responsables ni presupuestos asignados:

\begin{abstract}
Esta Instancia llama la atención sobre la existencia de puntos en los Acuerdos que están avanzando en la implementación sin los enfoques de género y diferencial. Tal es el caso de los PDET, los Pisda y los PNIS. Solicitamos que los contenidos e indicadores del punto 4 sean revisados pues corresponde a actividades o se presentan como neutrales al género. (p. 11)
\end{abstract}

En cuanto al borrador del Plan Marco para la Implementación (PMI) del Acuerdo de Paz, esta Instancia Especial advierte en su momento lo siguiente:

Esta Instancia considera que el Gobierno Nacional debe entregar para su análisis todo el plan 
marco y no sólo [sic] el capítulo de género. Puesto que como se reitera a lo largo de los acuerdos, su implementación debe garantizar de manera transversal los enfoques de derechos, género y diferencial. Así mismo no es conveniente tener un capítulo específico que desarrolle el tema de género, porque tiende a reproducir la idea que éste [sic], es algo que se agrega y no estructural al análisis y eliminación de la discriminación a través de las políticas, y reduce la idea de la transversalidad del enfoque. Por ello consideramos que la revisión que hace esta Instancia debe ser sobre la totalidad del PMI. (2018, p. 12)

Finalmente, la Instancia Especial señala las dificultades que se tuvieron para su funcionamiento debido a la ausencia de recursos económicos y humanos para facilitar sus tareas de apoyo a la Comisión de Seguimiento, Impulso y Verificación a la Implementación del Acuerdo Final (CSIVI).

En tercer lugar, se tiene como referente el informe N. 5 , fechado el 5 de enero del 2018, del Observatorio de seguimiento a la Implementación del Acuerdo de Paz (OIAP), titulado La Paz en Deuda, el cual señala que a 2018 , solo se ha implementado el 18,3\% del Acuerdo de Paz, además, frente al balance legislativo, el Congreso solo ha aprobado 10 de 24 proyectos de ley que correspondían a la vigencia. El Observatorio estima que son cerca de "95 normas, entre reformas constitucionales, legales y decretos para asegurar una implementación integral"
(2018, p. 5). Adicionalmente, consideran

lo siguiente como un desacierto:

El trámite de las reformas en el Congreso del Fast Track fue lento, y tortuoso y el legislativo no respondió a la urgencia de adecuar un marco normativo para la paz, el Gobierno no termina de ajustar la institucionalidad y el presupuesto para cumplir lo acordado y algunos fallos de la Corte Constitucional envían mensajes contradictorios en torno al espíritu del pacto, mientras crecen las disidencias de las FARC-EP y el asesinato de excombatientes y líderes sociales. (2018, p. 6)

Ahora bien, en cuanto al avance en la implementación del enfoque de género, el OIAP afirma:

\begin{abstract}
En el caso del enfoque de género, salvo el Decreto de vivienda social rural que establece que el acceso a las soluciones de vivienda será equitativo, que las mujeres tendrán una participación activa en la definición y ejecución del plan y que uno de los criterios para la priorización de subsidios familiares de vivienda será el de mujeres cabeza de familia y madres comunitarias, las demás disposiciones no cuentan con disposiciones que garanticen la priorización del acceso a mujeres pese a que fue lo acordado. (p. 18)
\end{abstract}

Por otro lado, con respecto a la implementación de los puntos del Acuerdo, señalan que "a la débil implementación de los puntos uno y cuatro se suman el desconocimiento de los enfoques diferen- 
ciales étnicos y de género contemplados en el Acuerdo Final" (p. 20), y en cuanto al atraso en la adopción de normas y mecanismos correspondientes al punto seis del Acuerdo, enfatizan en la ausencia del enfoque de género: "pero esos atrasos e incumplimientos son más evidentes, cuando se trata del componente étnico y de enfoque de género" (p. 57).

Finalmente, se mencionan los informes de la Comisión Internacional de Verificación de los Derechos Humanos en Colombia, la cual integrada por organizaciones sociales y cuenta con el apoyo de personas e instituciones de Alemania, Bélgica, Portugal, Francia, Colombia y España. Se estudió el Informe de febrero del 2018 sobre la verificación de los Derechos Humanos en la Comunidad de Paz de San José de Apartadó centrado en: "Realizar un seguimiento de la implementación de dichos acuerdos, para detectar incumplimientos que puedan llevar a la situación de vulneración y violaciones de DDHH, como sucedió en anteriores Acuerdos de Paz." (p. 16), así como en visibilizar la situación de vulneración de los derechos humanos en la Comunidad de Paz de San José de Apartadó (p. 16). Durante el 2017, en dicha comunidad se observó el incremento de fuerzas paramilitares y agresiones a los líderes comunitarios (pp. 20-35). Adicionalmente, frente al Acuerdo de Paz y el tema de género señaló lo siguiente:

Manifestamos nuestra preocupación por la situación de las mujeres ya que en el segundo acuerdo firmado (tras el plebisci- to del 2 de octubre de 2016), en este nuevo proceso de justicia para la Paz, han retrocedido en consecución de derechos, como lo relativo a la participación de las mujeres en la vida política a partir del concepto de paridad, o como la consecución de los derechos de la población LGTB, que, en el actual acuerdo, casi no tiene presencia. (p. 67)

Esta situación de retroceso en términos de garantías reales que permitan materializar la equidad y la paridad en la participación política surge de la redacción inadecuada de las normas, la ausencia de acciones afirmativas concretas y de formación jurídico-política a las comunidades que aún experimentan el miedo y las discriminaciones. Adicionalmente, derivado de sus constataciones en la Comunidad de Paz de San José de Apartadó:

$$
\begin{aligned}
& \text { Mostramos nuestra preocupación } \\
& \text { en la violencia cotidiana que se } \\
& \text { ejerce contra las mujeres, por el } \\
& \text { derecho a pensar y decidir, que, } \\
& \text { aunque la ley } 1257 \text { sobre la no } \\
& \text { violencia contra las mujeres existe } \\
& \text { a nivel nacional, la protección } \\
& \text { de las mujeres en su vida diaria } \\
& \text { requiere una implantación mayor } \\
& \text { por parte del estado. Se nos } \\
& \text { relató la cifra que dibuja esta } \\
& \text { realidad, en la que, de } 193 \text { casos } \\
& \text { de violencia sexual, solo } 3 \text { casos } \\
& \text { fueron condenados. (p. } 67 \text { ) }
\end{aligned}
$$

Como se puede observar, los tres primeros informes se centran en la verificación de la implementación desde un punto de visto normativo-político y coinciden en que la implementación del Acuerdo 
de Paz, transversalizado por enfoques de género, aún es incipiente y no se ha logrado lo propuesto. El cuarto informe que realiza una constatación en campo muestra la imposibilidad y la falta de conocimiento que tienen las instancias e instituciones territoriales para hacer frente a las realidades que en la cotidianidad ahondan las diferencias, discriminación y violencias frente a las mujeres.

\section{PLAN MARCO PARA LA IMPLEMENTACIÓN DEL ACUERDO DE PAZ}

Para efectos de este artículo, se hace referencia al Plan Marco para la Implementación del Acuerdo de Paz (PMI) brevemente, en tanto obedece a la expresión de una política de largo plazo, en el entendido de que la articulación entre las instituciones -en términos de recursos financieros y humanos- es necesaria para avanzar en la implementación. El PMI fue expedido por el Gobierno Nacional y la CSIVI con miras a los próximos 15 años y se complementa con el Conpes 3932 Lineamientos para la articulación del Plan Marco de Implementación del Acuerdo Final, con los instrumentos de planeación, programación y seguimiento a políticas públicas del orden nacional y territorial, expedido el 28 de junio del 2018.

En el contenido del PMI vale la pena resaltar el capítulo II "Enfoques transversales de género y étnico", en el cual se establecen: 1) los principios orientadores para incorporar de forma transversal el enfoque de género en el Acuerdo de Paz; 2) la ruta de transversalización del enfo- que de género durante la implementación del Acuerdo de Paz; 3) el diagnóstico; y 4) las estrategias e indicadores para la transversalización del enfoque de género en la implementación del Acuerdo Final, que incluye 51 indicadores de género. En este sentido, al momento de formular normas y políticas públicas y dar aplicación a la normatividad existente, cobra importancia tener presentes los siguientes principios rectores: paridad, integralidad, participación, seguridad humana, interseccionalidad y corresponsabilidad.

Finalmente, está el Decreto 1418 del 3 de agosto de 2018, por el cual se crea la Comisión Intersectorial para la incorporación del enfoque de género en la implementación del Acuerdo final para la terminación del conflicto y la construcción de una paz estable y duradera, la cual se denominará Alta Instancia de Género de Gobierno. Esta estará conformada por: director/a del Departamento Administrativo de la Presidencia de la República, ministro/a del Interior, ministro/a de Hacienda, ministro/a de Agricultura y Desarrollo Rural, director/a del Departamento Nacional de Planeación, director/a del Departamento Administrativo de la Función Pública, director/a de la Agencia de Renovación del Territorio y director/a de la Agencia para la Reincorporación y Normalización. Asimismo, señalan que "La Secretaría Técnica de la alta Instancia de Género de Gobierno, será ejercida por el Departamento Administrativo de la Presidencia (Consejería Presidencial para la Equidad de la Mujer)" (Presidencia, 
2018, art.8) - la cual pasó de ser Alta Consejería para la Equidad de la Mujer a Consejería Presidencial para la Equidad de la Mujer mediante el Decreto 672 de 26 de abril 2017-, con lo que se adicionan más funciones a las ya existentes, entre ellas las de preparar el reglamento interno de esta nueva comisión y proponer mecanismos para la implementación y monitoreo.

Adicionalmente, uno de los cuestionamientos frente a este Decreto, por ejemplo cuando se nombra a los integrantes de la Alta Instancia de Género de Gobierno, es que utilizan expresiones tales como: "el/ la ministro/a", como si el uso de artículos o sustantivos femeninos fuera suficiente para entender la incorporación de un enfoque de género y se dejan de lado principios como el de la paridad. Es decir, para avanzar en la implementación con enfoque de equidad de género para la mujer, se tendría que garantizar que el 50\% de personas que conforman la comisión sean mujeres que efectivamente impulsen y den directrices y lineamientos para cristalizar la aspiración de que hombres y mujeres efectivamente tengan iguales oportunidades y acceso a derechos y garantías en tanto seres humanos, esto para señalar que al constatar la composición actual de dichos cargos, se advierte que el $75 \%$ son hombres.

Así las cosas, se hace evidente que en el texto final del Acuerdo de Paz, cada que se menciona el enfoque de género, se trata de forma diferente, en algunos puntos habla de "mujeres", en otros es más amplio, en otros más restrictivo, pero dicha variedad de formas de nombrar se trasladan a los diversos documentos, note el lector la diferencia, por ejemplo, en el PMI que señala:
El Acuerdo Final para la termi- nación del conflicto y la cons- trucción de una paz estable y duradera, definió el enfoque de género como "el reconocimien- to de la igualdad de derechos entre hombres y mujeres y de las circunstancias especiales de cada uno, especialmente de las mujeres independientemente de su estado civil, ciclo vital, relación familiar y comunitaria, como sujeto de derechos y de especial protección constitucional. Esto implica en particular la necesidad de garantizar medidas afirmativas para promover esa igualdad, la participación activa de las mujeres y sus organizaciones en la construcción de la paz y el reconocimiento de la victimi- zación de la mujer por causa del conflicto". (Gobierno de Colombia y FARC-EP, 2016, p. 194). (PMI, 2018, p. 144)

En contraste, en documentos tales como el Conpes 3931 de 2018 Política Nacional para la Reincorporación Social y Económica de exintegrantes de las FARC-EP, al referirse al enfoque de género señalan:
Es el reconocimiento de las muje- res como ciudadanas autónomas, sujetos de derechos que, inde- pendientemente de su estado civil, relación familiar o comuni- taria, con acceso en condiciones de igualdad con respecto a los hombres a la propiedad de la 
tierra y proyectos productivos, opciones de financiamiento, infraestructura, servicios técnicos y formación, entre otros; atendiendo las condiciones sociales e institucionales que han impedido a las mujeres acceder a activos productivos y bienes públicos y sociales. Este reconocimiento implica la adopción de medidas específicas en la planeación, ejecución y seguimiento a los planes y programas contemplados en este Acuerdo para que se implementen teniendo en cuenta las necesidades específicas y condiciones diferenciales de las mujeres, de acuerdo con su ciclo vital, afectaciones y necesidades. (DNP, 2018a, p. 32)

Estas diferencias al nombrar no son pacíficas y no son propias del Acuerdo, detrás de cada manera existe un acervo teórico que identifica diferentes corrientes feministas, quizás aún no tenemos elementos para establecer un consenso sobre el alcance de la implementación del Acuerdo de Paz desde una perspectiva de género.

\section{CONCLUSIONES}

A manera de recapitulación, es importante entender que género no significa feminismo, ni mujer, ni población LGBTIO, y que muchas propuestas o estudios que van dirigidos a garantizar derechos en los que se abordan las violencias que sufren las mujeres, niños (independientemente de su género) y grupos LGBTIQ, no necesariamente están elaborados desde un enfoque de género y mucho menos desde un enfoque de equidad de género.
La categoría género busca cuestionar los roles que se han asignado a hombres y mujeres a lo largo de la historia y en las diferentes regiones geográficas, las cuales han contribuido a una desigualdad estructural entre hombres y mujeres basados en su sexo, orientaciones sexuales o identidades sexuales, las cuales influyen decisivamente en la asignación de derechos y garantías por parte del Estado, la sociedad y los mismos operadores del derecho al ejercer sus funciones de regulación, aplicación, interpretación, disciplinamiento e incluso acompañamiento de la satisfacción de los derechos humanos de todo el conjunto de la población colombiana. Estos derechos se deben entender como inherentes a la dignidad humana.

Las razones anteriores justifican avanzar en este tipo de proyectos puesto que de un lado se advierte la lenta expedición de normas que permitan materializar los acuerdos y de otro lado, algunas de ellas carecen de un verdadero enfoque de equidad de género, conceptos como "enfoques territoriales, diferenciados y de género" se volvieron muletillas carentes de contenido teórico y práctico que permitan materializar efectivamente el derecho a la paz y eliminar las discriminaciones en los ámbitos públicos y privados de los cuales son objeto/sujeto las mujeres.

Así las cosas, se hace necesario identificar los matices utilizados al nombrar y dotar de significado el enfoque de género, es decir, de qué hablamos en Colombia como sociedad, a qué se refería el Acuerdo de 
Paz, de qué hablan las instituciones políticas y administrativas en sus territorios, qué enseñamos en nuestras aulas cuando decimos que es necesario transversalizar el "enfoque de género". Dicho análisis será objeto de otro artículo, puesto que será necesario identificar las corrientes teóricas feministas y las conquistas de los movimientos sociales que permitan clarificar en términos conceptuales para posteriormente establecer los indicadores que permitan cuantificar y verificar la presencia de principios como: equidad, paridad e interseccionalidad, no solo en el ámbito normativo, sino en el avance de la transformación de las instituciones.

\section{REFERENCIAS}

Alta Consejería Presidencial para la Equidad de la Mujer (Acpem) (2012) Lineamientos para la Política Pública de Equidad de Género para las Mujeres. Bogotá, Colombia: Presidencia de la República de Colombia. Recuperado de: http://www.equidadmujer.gov.co/ Documents/Lineamientos-politica-publicaequidad-de-genero.pdf

Alto Comisionado para la Paz (2016) La paz es conmigo: Las mujeres como protagonistas en la construcción de paz. Bogotá, Colombia. Recuperado de: http://www.altocomisionadoparalapaz. gov.co/herramientas/Documents/Cartillamujeres-paz-12-sept-2016.pdf

Asamblea Nacional Constituyente, ANC (1991). Constitución Política de Colombia. Bogotá, Colombia. Recuperado de: http://wsp. presidencia.gov.co/Normativa/Documents/ Constitucion-Politica-Colombia.pdf
Cobos Bedia, R. (2014). Aproximaciones a la teoría crítica feminista. Lima, Perú: Cladem. Recuperado de http://www.cladem.org/pdf/ Aproximaciones-a-la-teoria-critica.pdf

Comisión Internacional de Verificación de los Derechos Humanos en Colombia (2018), Informe Comunidad de Paz de San José de Apartadó. Recuperado de http://iueuropa.org/ wp-content/uploads/2018/02/Informede-la-Comisi\%C3\%B3n-Internacional-deVerificaci\%C3\%B3n-de-Derechos-Humanosen-Colombia.pdf

Comité para la Eliminación de la Discriminación contra la Mujer (2013, octubre 29). Observaciones finales sobre los informes periódicos séptimo y octavo combinados de Colombia. Recuperado de http://historico.equidadmujer.gov.co/ Documents/Observaciones-CEDAW-VII-VIIIInforme-Colombia.pdf

Congreso de la República (1981). Ley 51 de 1981. Por medio de la cual se aprueba la "Convención sobre la eliminación de todas las formas de discriminación contra la mujer", adoptada por la Asamblea General de las Naciones Unidas el 18 de diciembre de 1979 y firmada en Copenhague el 17 de julio de 1980. Diario Oficial (35.794). Recuperado de https://www.alcaldiabogota.gov.co/sisjur/ normas/Normal.jsp?i=14153

Congreso de la República (1995). Ley 248 de 1995. Por medio de la cual se aprueba la Convención Internacional para prevenir, sancionar y erradicar la violencia contra la mujer, suscrita en la ciudad de Belem Do Para, Brasil, el 9 de junio de 1994. Diario Oficial (42.171). Recuperado de https://www.alcaldiabogota. gov.co/sisjur/normas/Normal.jsp?i=37821

Congreso de la República (1996). Ley 294 de 1996. Por la cual se desarrolla el artículo 42 de la Constitución Política y se dictan normas para prevenir, remediar y sancionar la violencia intrafamiliar. Diario Oficial (42.836). Recuperada 
de https://www.icbf.gov.co/cargues/avance/ docs/ley_0294_1996.htm

Congreso de la República (2008). Ley 1257 de 2008. Por la cual se dictan normas de sensibilización, prevención y sanción de formas de violencia y discriminación contra las mujeres, se reforman los Códigos Penal, de Procedimiento Penal, la Ley 294 de 1996 y se dictan otras disposiciones. Diario Oficial (47.193). Recuperada de https://www.oas.org/ dil/esp/LEY_ 1257 _DE_2008_Colombia. $\underline{\mathrm{pdf}}$

Congreso de la República (2011). Ley 1450 de 2011. Por la cual se aprueba el Plan de Desarrollo 2010-2014. Diario Oficial (48.101). Recuperado de https://www.procuraduria.gov.co/portal/ media/file/docs/ddr/CompiladoNormativo _ Parte3.pdf

Congreso de la República (2015). Ley 1753 de 2015. Plan Nacional de Desarrollo 2015-2018. Diario Oficial (49.538). Recuperada de http:// www.secretariasenado.gov.co/senado/ basedoc/ley_1753_2015.html

Denzin, N. y Lincoln Y. (2012) Controversias paradigmáticas, contradicciones y confluencias emergentes. En N. Denzin y Y. Lincoln (Coords.), El campo de la investigación Cualitativa. Manual de investigación cualitativa (vol. I, pp. 43-102). Barcelona: Gedisa.

Departamento Nacional de Planeación, DNP (2013). Conpes 161. Política Pública de Equidad de Género para las Mujeres. Recuperado de: https:// colaboracion.dnp.gov.co/CDT/Conpes/ Social/161.pdf

Departamento Nacional de Planeación, DNP (2018a). Conpes 3931 Política Nacional para la Reincorporación Social y Económica de exintegrantes de las FARC-EP. Recuperado de https:// colaboracion.dnp.gov.co/CDT/Conpes/ Econ\%C3\%B3micos/3931.pdf

Departamento Nacional de Planeación, DNP (2018b). Conpes 3932 Lineamientos para la
Articulación del Plan Marco de Implementación del Acuerdo Final, con los instrumentos de planeación, programación y seguimiento a políticas públicas del orden nacional $y$ territorial. Recuperado de https://colaboracion.dnp.gov.co/CDT/ Conpes/Económicos/3932.pdf

El Tiempo. Redacción Judicial (7 marzo 2018) Freno del Consejo de Estado a la Comisión de Seguimiento del Acuerdo de Paz. Recuperado de https://www.elespectador.com/noticias/ judicial/freno-del-consejo-de-estado-lacomision-de-seguimiento-del-acuerdo-depaz-articulo-743003

Facio Montejo, A. (1992). Cuando el género suena cambios trae (Una metodología para el análisis de género del fenómeno legal). San José, C.R.: Ilanud.

Galtung, J. (2009, octubre-noviembre). Equidad, un principio para construir la paz: Galtung. Hechos del Callejón 5(50), 13-15.

Gobierno de Colombia y FARC-EP (2016). Acuerdo final para la terminación del conflicto y la construcción de una paz estable y duradera. Bogotá, Colombia: República de Colombia y FARC-EP. Recuperado de http://www. altocomisionadoparalapaz.gov.co/procesos-y-conversaciones/Documentos\%20 compartidos/24-11-2016NuevoAcuerdoFinal. $\underline{\mathrm{pdf}}$

Gobierno Nacional (2017). Plan Marco para la Implementación de los Acuerdos de Paz. Recuperado de: https://colaboracion.dnp.gov.co/ CDT/Conpes/Econ\%C3\%B3micos/3932 Anexo\%20B_Plan\%20Marco\%20de\%20 Implementaci\%C3\%B3n\%20(PMI).pdf

Grupo de Trabajo el Género en Paz (GPAZ). (2017, agosto 16). Referencia: Intervención de GPAZ en la Audiencia Pública en el marco de la revisión oficiosa de constitucionalidad del decreto 902 de 2017 "Por el cual se adoptan medidas para facilitar la implementación de la Reforma Rural Integral contemplada en el Acuerdo Final en materia de tierras, específicamente el procedimiento para el acceso y 
Marco de referencia para reflexionar sobre la implementación del Acuerdo de Paz, desde el enfoque...

formalización y el Fondo de Tierras". Recuperado el 8 de Septiembre de 2017, Recuperado de https://www.dejusticia.org/wp-content/ uploads/2017/08/INTERVENCIO\%CC\%81NAUDIENCIA-902-GPAZ-.pdf-2-1-Final.pdf

Instancia Especial para contribuir a garantizar el enfoque de género en la implementación del Acuerdo Final (2018) Primer Informe de Gestión de la Instancia Especial de Género 2017-2. Recuperado de https://csivi. co/sites/default/files/2018-05/Primer\%20 Informe\%20Gesti\%C3\%B3n\%20Instancia\%20 Especial\%20Julio-Diciembre\%202017\%20 FINAL.pdf

Instituto Kroc de Estudios Internacionales de Paz (2017). Informe sobre el estado efectivo de implementación del acuerdo de paz en Colombia. Notre Dame: Universidad de Notre Dame. Recuperado de http://static.iris.net.co/ semana/upload/documents/informe-kroc.pdf

Instituto Kroc de Estudios Internacionales de Paz (2018). Segundo informe sobre el estado efectivo de Implementación del Acuerdo de Paz en Colombia diciembre 2016 - mayo 2018. Notre Dame: Universidad de Notre Dame. Recuperado de https://kroc.nd.edu/assets/284864/informe_2_instituto_kroc_final_with_logos.pdf

Lopera, M.., Roldán, J., Cardona, E., Álvarez, S., Arcila, M., Aguirre, J. y Mendoza, M. (2018, abril-junio). La reforma rural integral en el acuerdo final para la terminación del conflicto y la construcción de una paz estable y duradera desde el enfoque de equidad de género para las mujeres. Diálogos de Derecho y Política, (20), 42-67. Recuperado de https://aprendeenlinea.udea.edu.co/ revistas/index.php/derypol/article/download/332510/20788403

Mazzoldi, G., y Cuesta, I. (2017, febrero 2). Debates en torno al enfoque de género en el acuerdo de paz colombiano. Open Democracy. Recuperado de https://www.opendemocracy. net/es/debates-transformaciones-y-potencialidades-del-enfoqu/

Observatorio de Seguimiento a los Acuerdos de Paz (OIAP) (2018). La Paz en Deuda, Informe No. 5. Recuperado de: https://oiapblog.files. wordpress.com/2018/01/la-paz-en-deuda.pdf

ONU (1979) Convención sobre la Eliminación de Todas las Formas de Discriminación contra las Mujeres. Recuperado de https://www.un.org/womenwatch/daw/cedaw/text/sconvention.htm

ONU Mujeres (s.f.). Incorporación de la perspectiva de género. Recuperado de: http://www.unwomen. org/es/how-we-work/un-system-coordination/gender-mainstreaming

Organización de los Estados Americanos (OEA) (1995, agosto 14). Convención Interamericana para Prevenir, Sancionar y Erradicar la Violencia contra la Mujer, (Convención de Belem do Pará). Washington: OEA. Recuperado de https:// www.oas.org/dil/esp/convencion_belem_do_para.pdf

Organización Internacional para las Migraciones (OIM) (2018). Spotlight: Género y la Implementación del Acuerdo de Paz en Colombia. Parte I. Recuperado de http://iueuropa. org/wp-content/uploads/2018/02/Informede-la-Comisi\%C3\%B3n-Internacional-deVerificaci\%C3\%B3n-de-Derechos-Humanosen-Colombia.pdf

Pineda García, R. (2017, octubre 24). Conversatorio Acuerdos de Paz con enfoque de Equidad de Género para las Mujeres ¿Y de la implementación qué? Facultad de Derecho y Ciencias Políticas, Universidad de Antioquia, Medellín, Colombia.

Presidencia (2018, agosto 3). Decreto 1418 de 2018. Por el cual se crea la Comisión Intersectorial para la incorporación del enfoque de Género en la implementación del Acuerdo Final para la Terminación del Conflicto y la Construcción de una Paz Estable y Duradera, 


\section{Elvigia Cardona Zuleta}

la cual se denominará Alta Instancia de Género de Gobierno. Diario Oficial. Recuperada de: http://www.funcionpublica.gov.co/eva/ gestornormativo/norma.php?i=87871 culturales en clave de-colonial. Tabula Rasa, (12), 209-227.

Walsh, C. (2010, enero-junio). Estudios (inter) 